\title{
PROOFS FOR TWO LAMBERT SERIES IDENTITIES OF GOSPER
}

\author{
BING HE
}

\begin{abstract}
Applying the theory of modular forms and Lambert series manipulations we establish an Eisenstein series identity. From this formula we confirm a Lambert series identity conjectured by Gosper. Another Lambert series identity of Gosper is also confirmed by using Lambert series manipulations.
\end{abstract}

\section{INTRODUCTION}

In [3, pp. 102-103] Gosper conjectured the following three identities on Lambert series:

$$
\begin{aligned}
\sum_{n \geq 1} \frac{q^{2 n-1}}{\left(1-q^{2 n-1}\right)^{2}}-2 \sum_{n \geq 1} \frac{q^{4 n-2}}{\left(1-q^{4 n-2}\right)^{2}} & =\sum_{n \geq 1} \frac{(2 n-1) q^{2 n-1}}{1-q^{4 n-2}} \\
\sum_{n \geq 1} \frac{n q^{2 n}}{1+q^{2 n}} \sum_{n \geq 1} \frac{(2 n-1) q^{2 n-1}}{1-q^{4 n-2}} & =\sum_{n \geq 1} \frac{B_{3}(n)}{3} \frac{q^{2 n-1}}{1-q^{4 n-2}}
\end{aligned}
$$

and

$$
6 \sum_{n \geq 1} \frac{q^{4 n-2}}{\left(1-q^{2 n-1}\right)^{4}}+\sum_{n \geq 1} \frac{q^{2 n-1}}{\left(1-q^{2 n-1}\right)^{2}}=\sum_{n \geq 1} \frac{n^{3} q^{n}}{1-q^{2 n}},
$$

where

$$
B_{3}(n):=n(n-1)(n-1 / 2) .
$$

Both sides of these identities involve only Lambert series, but the second one is different from the other two formulas because the left side of (1.2) is a product of two Lambert series. It should be emphasized that there are no identities of this type in Gosper's list except these three formulas. Actually, the identity (1.1) was confirmed by El Bachraoui in [2, p. 7] while the other two formulas remain open.

In this paper we will confirm the Lambert series identities (1.2) and (1.3).

Theorem 1.1. The identities (1.2) and (1.3) hold for $|q|<1$.

In Section 2, we employ the theory of modular forms to establish an Eisenstein series identity, which is crucial in the derivation of (1.2). Section 3 is devoted to our proof of Theorem 1.1 .

2000 Mathematics Subject Classification. 11F11, 11M36.

Key words and phrases. Lambert series; Modular form; Eisenstein series. 


\section{AN AUXILIARY RESUlt}

We first recall some notations from the theory of modular forms.

The modular group $\mathrm{SL}_{2}(\mathbb{Z})$ is defined by

$$
\mathrm{SL}_{2}(\mathbb{Z}):=\left\{\left(\begin{array}{ll}
a & b \\
c & d
\end{array}\right): a, b, c, d \in \mathbb{Z}, a d-b c=1\right\} .
$$

If $N>1$ is an integer, the congruence subgroup $\Gamma_{0}(N)$ is defined by

$$
\Gamma_{0}(N)=\left\{\left(\begin{array}{ll}
a & b \\
c & d
\end{array}\right) \in \mathrm{SL}_{2}(\mathbb{Z}): c \equiv 0(\bmod N)\right\} .
$$

We denote by $M_{k}(\Gamma)$ the space of modular forms of weight $k$ for $\Gamma$ if $\Gamma$ is a subgroup of $\mathrm{SL}_{2}(\mathbb{Z})$ with finite index.

In order to prove (1.2) we need an auxiliary result.

Lemma 2.1. For $\operatorname{Im} \tau>0$, we have

$$
\begin{aligned}
& E_{4}(\tau)-9 E_{4}(2 \tau)+8 E_{4}(4 \tau) \\
& =10\left(2 E_{2}(4 \tau)-E_{2}(2 \tau)\right)\left(3 E_{2}(2 \tau)-E_{2}(\tau)-2 E_{2}(4 \tau)\right),
\end{aligned}
$$

where $E_{2}(\tau)$ and $E_{4}(\tau)$ are two Eisenstein series given by

$$
\begin{aligned}
& E_{2}(\tau):=1-24 \sum_{n \geq 1} \sigma_{1}(n) e^{2 \pi n i \tau}, \\
& E_{4}(\tau):=1+240 \sum_{n \geq 1} \sigma_{3}(n) e^{2 \pi n i \tau}
\end{aligned}
$$

with

$$
\sigma_{\alpha}(n):=\sum_{d \mid n} d^{\alpha} .
$$

Proof. It follows from [1, Exercise 1.2.8(e)] that

$$
E_{2}(\tau)-N E_{2}(N \tau) \in M_{2}\left(\Gamma_{0}(N)\right) .
$$

In particular,

$$
E_{2}(\tau)-2 E_{2}(2 \tau) \in M_{2}\left(\Gamma_{0}(2)\right)
$$

Then

$$
2 E_{2}(4 \tau)-E_{2}(2 \tau) \in M_{2}\left(\Gamma_{0}(4)\right)
$$

and

$3 E_{2}(2 \tau)-E_{2}(\tau)-2 E_{2}(4 \tau)=2 E_{2}(2 \tau)-E_{2}(\tau)+E_{2}(2 \tau)-2 E_{2}(4 \tau) \in M_{2}\left(\Gamma_{0}(4)\right)$.

Therefore, the right side of (2.1) belongs to $M_{4}\left(\Gamma_{0}(4)\right)$. Since $E_{4}(\tau) \in M_{4}\left(\mathrm{SL}_{2}(\mathbb{Z})\right.$ ), we see that $E_{4}(2 \tau) \in M_{4}\left(\Gamma_{0}(2)\right)$ and $E_{4}(4 \tau) \in M_{4}\left(\Gamma_{0}(4)\right)$. From [1, Theorem 3.5.1] we know that $\operatorname{dim}\left(M_{4}\left(\Gamma_{0}(4)\right)\right)=3$. It is clear that $\left\{E_{4}(\tau), E_{4}(2 \tau), E_{4}(4 \tau)\right\}$ is a basis of $M_{4}\left(\Gamma_{0}(4)\right)$. Then there exist three complex numbers $a, b, c$ such that

$$
\begin{aligned}
& 10\left(2 E_{2}(4 \tau)-E_{2}(2 \tau)\right)\left(3 E_{2}(2 \tau)-E_{2}(\tau)-2 E_{2}(4 \tau)\right) \\
& =a E_{4}(\tau)+b E_{4}(2 \tau)+c E_{4}(4 \tau) .
\end{aligned}
$$

Set $q=e^{2 \pi i \tau}$. Comparing the coefficients of $q^{0}, q^{1}, q^{2}$ on both sides of (2.2) we get

$$
a=1, b=-9, c=8 .
$$

Then (2.1) follows readily by substituting these constants back into (2.2). 


\section{Proof of Theorem 1.1}

We first prove (1.2). Since

$$
\begin{aligned}
& \frac{q^{2 n}}{1+q^{2 n}}=\frac{q^{2 n}}{1-q^{2 n}}-\frac{2 q^{4 n}}{1-q^{4 n}}, \\
& \frac{q^{n}}{1-q^{2 n}}=\frac{q^{n}}{1-q^{n}}-\frac{q^{2 n}}{1-q^{2 n}} .
\end{aligned}
$$

we see that

$$
\begin{gathered}
\sum_{n \geq 1} \frac{n q^{2 n}}{1+q^{2 n}}=\sum_{n \geq 1} \frac{n q^{2 n}}{1-q^{2 n}}-2 \sum_{n \geq 1} \frac{n q^{4 n}}{1-q^{4 n}}, \\
\sum_{n \geq 1} \frac{n q^{n}}{1-q^{2 n}}=\sum_{n \geq 1} \frac{n q^{n}}{1-q^{n}}-\sum_{n \geq 1} \frac{n q^{2 n}}{1-q^{2 n}}
\end{gathered}
$$

and

$$
\sum_{n \geq 1} \frac{n q^{2 n}}{1-q^{4 n}}=\sum_{n \geq 1} \frac{n q^{2 n}}{1-q^{2 n}}-\sum_{n \geq 1} \frac{n q^{4 n}}{1-q^{4 n}} .
$$

From the last two identities above we deduce that

$$
\begin{aligned}
\sum_{n \geq 1} \frac{(2 n-1) q^{2 n-1}}{1-q^{4 n-2}} & =\sum_{n \geq 1} \frac{n q^{n}}{1-q^{2 n}}-\sum_{n \geq 1} \frac{2 n q^{2 n}}{1-q^{4 n}} \\
& =\sum_{n \geq 1} \frac{n q^{n}}{1-q^{n}}-3 \sum_{n \geq 1} \frac{n q^{2 n}}{1-q^{2 n}}+2 \sum_{n \geq 1} \frac{n q^{4 n}}{1-q^{4 n}} .
\end{aligned}
$$

Similarly,

$$
\begin{aligned}
& \sum_{n \geq 1} \frac{B_{3}(n)}{3} \frac{q^{2 n-1}}{1-q^{4 n-2}} \\
& =\frac{1}{24} \sum_{n \geq 1}(2 n-1)\left((2 n-1)^{2}-1\right) \frac{q^{2 n-1}}{1-q^{4 n-2}} \\
& =\frac{1}{24} \sum_{n \geq 1} n\left(n^{2}-1\right) \frac{q^{n}}{1-q^{2 n}}-\frac{1}{12} \sum_{n \geq 1} n\left(4 n^{2}-1\right) \frac{q^{2 n}}{1-q^{4 n}} \\
& =\frac{1}{24} \sum_{n \geq 1} n\left(n^{2}-1\right) \frac{q^{n}}{1-q^{n}}-\frac{1}{24} \sum_{n \geq 1} n\left(n^{2}-1\right) \frac{q^{2 n}}{1-q^{2 n}} \\
& -\frac{1}{12} \sum_{n \geq 1} n\left(4 n^{2}-1\right) \frac{q^{2 n}}{1-q^{2 n}}+\frac{1}{12} \sum_{n \geq 1} n\left(4 n^{2}-1\right) \frac{q^{4 n}}{1-q^{4 n}} \\
& =\frac{1}{24} \sum_{n \geq 1} n\left(n^{2}-1\right) \frac{q^{n}}{1-q^{n}}-\frac{1}{8} \sum_{n \geq 1}\left(3 n^{3}-n\right) \frac{q^{2 n}}{1-q^{2 n}} \\
& +\frac{1}{12} \sum_{n \geq 1} n\left(4 n^{2}-1\right) \frac{q^{4 n}}{1-q^{4 n}} .
\end{aligned}
$$


Let $q=e^{2 \pi i \tau}$ with $\operatorname{Im} \tau>0$. We rewrite the Eisenstein series $E_{2}(\tau)$ and $E_{4}(\tau)$ as

$$
\begin{aligned}
& E_{2}(\tau)=1-24 \sum_{n \geq 1} \frac{n q^{n}}{1-q^{n}}, \\
& E_{4}(\tau)=1+240 \sum_{n \geq 1} \frac{n^{3} q^{n}}{1-q^{n}} .
\end{aligned}
$$

Then

$$
\begin{aligned}
& \sum_{n \geq 1} \frac{n q^{n}}{1-q^{n}}=\frac{1}{24}\left(1-E_{2}(\tau)\right), \\
& \sum_{n \geq 1} \frac{n^{3} q^{n}}{1-q^{n}}=\frac{1}{240}\left(E_{4}(\tau)-1\right) .
\end{aligned}
$$

Substituting these identities into (3.1), (3.2) and (3.3) and then simplifying we get

$$
\begin{gathered}
\sum_{n \geq 1} \frac{n q^{2 n}}{1+q^{2 n}}=\frac{1}{24}\left(2 E_{2}(4 \tau)-E_{2}(2 \tau)\right)-\frac{1}{24}, \\
\sum_{n \geq 1} \frac{(2 n-1) q^{2 n-1}}{1-q^{4 n-2}}=\frac{1}{24}\left(3 E_{2}(2 \tau)-E_{2}(\tau)-2 E_{2}(4 \tau)\right), \\
\sum_{n \geq 1} \frac{B_{3}(n)}{3} \frac{q^{2 n-1}}{1-q^{4 n-2}}=\frac{1}{5760}\left(E_{4}(\tau)-9 E_{4}(2 \tau)+8 E_{4}(4 \tau)\right) \\
+\frac{1}{24^{2}}\left(E_{2}(\tau)-3 E_{2}(2 \tau)+2 E_{2}(4 \tau)\right) .
\end{gathered}
$$

Then (1.2) follows easily by adding $\frac{1}{24^{2}}\left(E_{2}(\tau)-3 E_{2}(2 \tau)+2 E_{2}(4 \tau)\right)$ into both sides of (2.1) and then substituting (3.4), (3.5) and (3.6) into the resulting identity.

We now show (1.3). It follows easily from the formula

$$
\frac{1}{1-x}=\sum_{n \geq 0} x^{n},|x|<1
$$

that

$$
\frac{x}{(1-x)^{2}}=\sum_{n \geq 1} n x^{n},|x|<1 .
$$

Differentiating (3.7) with respect to $x$ and then multiplying the resulting identity by $x$ we get

$$
\sum_{n \geq 1} n^{2} x^{n}=\frac{x(1+x)}{(1-x)^{3}},|x|<1
$$

Differentiating this equation with respect to $x$ again and then multiplying the resulting identity by $x$ we arrive at

$$
\sum_{n \geq 1} n^{3} x^{n}=\frac{x^{3}+4 x^{2}+x}{(1-x)^{4}},|x|<1 .
$$

Then

$$
\sum_{m \geq 1} \frac{q^{3 m}+4 q^{2 m}+q^{m}}{\left(1-q^{m}\right)^{4}}=\sum_{m, n \geq 1} n^{3} q^{m n}=\sum_{n \geq 1} \frac{n^{3} q^{n}}{1-q^{n}}
$$


and so

$$
\begin{aligned}
6 \sum_{m \geq 1} \frac{q^{2 m}}{\left(1-q^{m}\right)^{4}} & =\sum_{m \geq 1} \frac{q^{3 m}+4 q^{2 m}+q^{m}}{\left(1-q^{m}\right)^{4}}-\sum_{m \geq 1} \frac{q^{m}}{\left(1-q^{m}\right)^{2}} \\
& =\sum_{n \geq 1} \frac{n^{3} q^{n}}{1-q^{n}}-\sum_{m \geq 1} \frac{q^{m}}{\left(1-q^{m}\right)^{2}}
\end{aligned}
$$

Similarly,

$$
6 \sum_{m \geq 1} \frac{q^{4 m}}{\left(1-q^{2 m}\right)^{4}}=\sum_{n \geq 1} \frac{n^{3} q^{2 n}}{1-q^{2 n}}-\sum_{m \geq 1} \frac{q^{2 m}}{\left(1-q^{2 m}\right)^{2}} .
$$

We combine these two identities to give

$$
\begin{aligned}
6 \sum_{n \geq 1} \frac{q^{4 n-2}}{\left(1-q^{2 n-1}\right)^{4}} & =6 \sum_{n \geq 1} \frac{q^{2 n}}{\left(1-q^{n}\right)^{4}}-6 \sum_{n \geq 1} \frac{q^{4 n}}{\left(1-q^{2 n}\right)^{4}} \\
& =\sum_{n \geq 1} \frac{n^{3} q^{n}}{1-q^{2 n}}-\sum_{m \geq 1} \frac{q^{2 m-1}}{\left(1-q^{2 m-1}\right)^{2}} .
\end{aligned}
$$

From this we deduce (1.3). This completes the proof of Theorem 1.1 .

\section{ACKNOWLEDGEMENT}

This work was partially supported by the National Natural Science Foundation of China (Grant No. 11801451) and the Natural Science Foundation of Hunan Province (Grant No. 2020JJ5682).

\section{REFERENCES}

[1] F. Diamond and J. Shurman, A first course in modular forms, Graduate texts in mathematics 228. Springer-Verlag, New York, 2005.

[2] M. El Bachraoui, On series identities of Gosper and integrals of Ramanujan theta function $\psi(q)$, Proc. Amer. Math. Soc. 147(10)(2019), 4451-4464.

[3] R.W. Gosper, Experiments and discoveries in $q$-trigonometry, in: F.G. Garvan, M.E.H. Ismail (Eds.), Symbolic Computation, Number Theory, Special Functions, Physics and Combinatorics, Kluwer, Dordrecht, Netherlands, 2001, pp.79-105.

School of Mathematics and Statistics, Central South University, Changsha 410083, Hunan, People's Republic of China

Email address: yuhe001@foxmail.com; yuhelingyun@foxmail.com 